\title{
COMPONENT FUNCTORS
}

\section{ROBERT DAVIS}

Introduction. Many concrete categories $Q$ admit, besides the usual forgetful functor, another such functor $V: Q \rightarrow S$ (where $\delta$ is the category of sets) which may reasonably be termed a "component functor." For example, let $M$ be a monoid and let $a$ be the category of sets on which $M$ acts on the left. If $M$ acts on $A$, let $\sim$ be the equivalence relation generated by $a \sim m a$ for $a \in A, m \in M$. Then the component functor $V$ takes $A$ to the set $A / \sim$ of connected components of $A$ under the action of $M$.

Many such functors are especially interesting in that they have right adjoints $U: S \rightarrow Q$ which are monadic (i.e., triplable). In this paper we show that this situation will arise whenever $a$ is comonadic over $\delta$, and exhibit some typical examples.

1. Statement of the theorem. Let $F=(F, \epsilon, \delta)$ be a comonad over $\delta$. We recall that this means that $F: \delta \rightarrow S$ is a functor and that $\epsilon: F \rightarrow 1_{S}$ and $\delta: F \rightarrow F^{2}=F \circ F$ are natural transformations such that $F \epsilon \circ \delta=\epsilon F \circ \delta=1_{F}$ and $F \delta \circ \delta=\delta F \circ \delta$. An algebra over $F$ is a pair $(A, \xi)$ consisting of a set $A$ and a map $\xi: A \rightarrow F A$ such that $\epsilon_{A} \circ \xi=1_{A}$ and $F(\xi) \circ \xi=\delta_{A} \circ \xi$. A homomorphism $f:(A, \xi) \rightarrow(B, \rho)$ is a map $f: A \rightarrow B$ such that $\rho \circ f=F(f) \circ \xi$. Let $a$ be the category of algebras over $F$.

We note that $a$ possesses coproducts, which are formed by disjoint union just as in the category of sets. For example, the coproduct of $(A, \xi)$ and $(B, \rho)$ is $(A \coprod B, \omega)$ where the map $\omega: A \amalg B \rightarrow F(A \coprod B)$ is the composition

$$
A \amalg B \stackrel{\xi \amalg \stackrel{\rho}{\rightarrow}}{\longrightarrow} F(A) \quad \amalg F(B) \stackrel{\gamma}{\rightarrow} F(A \coprod B)
$$

and $\gamma$ is defined by $\gamma \circ i_{F(A)}=F\left(i_{A}\right), \gamma \circ i_{F(B)}=F\left(i_{B}\right)$. If $X$ is any set, we shall denote by $X \cdot A$ the coproduct of $|X|$ copies of $A$.

Note also that $Q$ has a terminal object $T$. Using 1 to denote a fixed one-element set, we have $T=F(1)$ equipped with the map $\delta_{1}: T$ $\rightarrow F(T)$. Now we are ready to state the

Theorem. The functor $U: S \rightarrow$ defined by $U(X)=X \cdot T$ is monadic.

The left adjoint $V$ to $U$ is called the component functor associated with $a$.

Received by the editors March 27, 1969. 
2. Proof of the theorem. First we shall prove that $U$ has a left adjoint $V$, by appealing to the adjoint-functor theorem of Freyd [4]. Observe that a map $f:(A, \xi) \rightarrow X \cdot T$ is a homomorphism iff the diagram

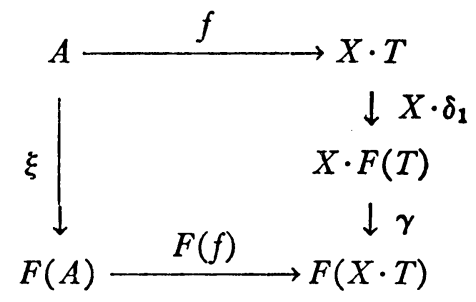

commutes. There is a unique homomorphism $e_{A}: A \rightarrow T$ which is equal to $F(\bar{e}) \circ \xi: A \rightarrow F(A) \rightarrow F(1)=T$ where $\bar{e}: A \rightarrow 1$ is the unique set map. Then for each $a \in A, f(a)$ must be the element $e_{A}(a)$ in some summand of $X \cdot T$ labeled by $\chi(a) \in X$. In this way we obtain a function $\chi: A$ $\rightarrow X$ and by chasing elements in the diagram it is easily seen that the diagram commutes iff for every $a \in A$, we have the additional property that

$$
F(f) \xi(a)=F\left(i_{\chi(a)}\right) \delta_{1} e_{A}(a) .
$$

Using this characterization of homomorphisms $A \rightarrow X \cdot T$ we shall show that the functor $U$ preserves limits.

To show that $U$ preserves equalizers, let $f, g: X \rightrightarrows Y$ and let $h: E$ $\rightarrow X$ be $e q(f, g)$. We claim that $h \cdot T: E \cdot T \rightarrow X \cdot T$ is the equalizer in $a$ of $f \cdot T$ and $g \cdot T$. For, suppose that $k:(A, \xi) \rightarrow X \cdot T$ is such that $(f \cdot T) \circ k=(g \cdot T) \circ k$. Then there is a unique set map $j: A \rightarrow E \cdot T$ such that $k=(h \cdot T) \circ j$, and we need only show that $j$ is a homomorphism. Let $k(a)$ be the element $e_{A}(a)$ in the summand of $X \cdot T$ labeled by $\chi(a)$ and let $j(a)$ be the element $e_{A}(a)$ in the summand of $E \cdot T$ labeled by $\eta(a)$. Then

$$
\begin{aligned}
F(h \cdot T) F(j) \xi(a) & =F(k) \xi(a) \\
& =F\left(i_{x(a)}\right) \delta_{1} e_{A}(a) \\
& =F(h \cdot T) F\left(i_{\eta(a)}\right) \delta_{1} e_{A}(a) .
\end{aligned}
$$

Since $F(h \cdot T)$ is monic, $F(j) \xi(a)=F\left(i_{\eta(a)}\right) \delta_{1} e_{A}(a)$ and thus $j$ is indeed a homomorphism.

Next, it is rather more difficult to show that $U$ preserves products; for simplicity we write the proof for binary products but the proof for infinitary products is similar. Thus we must prove that the product in $Q$ of $X \cdot T$ and $Y \cdot T$ is $(X \times Y) \cdot T$ where $X \times Y$ is the product 
in $\delta$. Suppose $f:(A, \xi) \rightarrow X \cdot T$ and $g:(A, \xi) \rightarrow Y \cdot T$, where $f(a)$ is the $e_{A}(a)$ in the $\chi(a)$-summand and $g(a)$ is the $e_{A}(a)$ in the $\eta(a)$-summand. Define $h: A \rightarrow(X \times Y) \cdot T$ by requiring $h(a)$ to be $e_{A}(a)$ in the summand labeled by $(\chi(a), \eta(a))$. Then clearly we need only show that $h$ is a homomorphism.

First, we note that

$$
\begin{aligned}
F(f) \xi(a) & =F\left(i_{x(a)}\right) \delta_{1} e_{A}(a) \\
& =F\left(i_{x(a)}\right) \delta_{1} F(\bar{e}) \xi(a) \\
& =F\left(i_{x(a)}\right) F^{2}(\bar{e}) \delta_{A} \xi(a) \\
& =F\left(i_{x(a)} \circ F(e) \circ \xi\right) \xi(a) \\
& =F\left(i_{x(a)} \circ e_{A}\right) \xi(a),
\end{aligned}
$$

and similarly $F(g) \xi(a)=F\left(i_{\eta(a)} \circ e_{\boldsymbol{A}}\right) \xi(a)$. To show that $h$ is a homomorphism, i.e., to show that the diagram

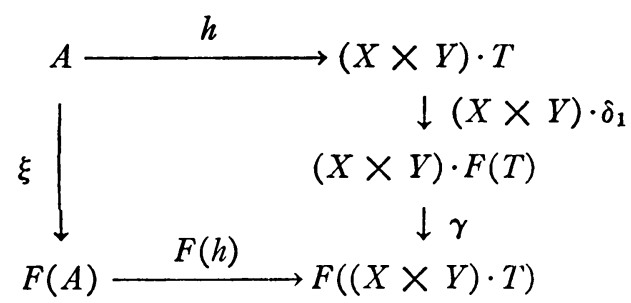

commutes, we must show that for all $a \in A$,

$$
F(h) \xi(a)=F\left(i_{(x(a), \eta(a))} \circ e_{A}\right) \xi(a) .
$$

The result would be immediate if we knew that the map $\left(F\left(p_{1}\right), F\left(p_{2}\right)\right)$ : $F((X \times Y) \cdot T) \rightarrow F(X \cdot T) \times F(Y \cdot T)$ is one-to-one. Unfortunately, there are many examples of comonads for which it is not one-to-one. However, it will suffice to show that $\left(F\left(p_{1}\right), F\left(p_{2}\right)\right)$ is one-to-one on the image of $\gamma \circ(X \times Y) \cdot \delta_{1}$. In fact, we shall show that the composition

$k=\left(F\left(p_{1}\right), F\left(p_{2}\right)\right) \circ \gamma \circ(X \times Y) \cdot \delta_{1}:(X \times Y) \cdot T \rightarrow F(X \cdot T) \times F(Y \cdot T)$ is actually a one-to-one function.

A little computation using the definition of $\gamma$ shows that $k$ $=\left(\gamma_{X}, \gamma_{Y}\right) \circ(X \times Y) \cdot \delta_{1}$, where $\gamma_{X}:(X \times Y) \cdot F(T) \rightarrow F(X \cdot T)$ is defined by $\gamma_{X} \circ i_{(x, y)}=F\left(i_{x}\right) . F(T) \rightarrow F(X \cdot T)$ and likewise $\gamma_{Y} \circ i_{(x, y)}=F\left(i_{y}\right)$. Then we have $k \circ i_{(x, y)}=\left(F\left(i_{x}\right), \quad F\left(i_{y}\right)\right) \circ \delta_{1}: T \rightarrow F(T) \rightarrow F(X \cdot T)$ $\times F(Y \cdot T)$. But $\delta_{1}, F\left(i_{x}\right)$, and $F\left(i_{y}\right)$ are one-to-one and hence $k \circ i_{(x, y)}$ is one-to-one for every $(x, y) \in X \times Y$. 
To show that $k$ is one-to-one, it now suffices to show that the images of distinct summands under $k$ are disjoint; that is, if $(x, y) \neq(\bar{x}, \bar{y})$ then the maps $\left(F\left(i_{x}\right), F\left(i_{y}\right)\right) \circ \delta_{1}$ and $\left(F\left(i_{\dot{x}}\right), F\left(i_{\bar{y}}\right)\right) \circ \delta_{1}: T \rightarrow F(X \cdot T)$ $\times F(Y \cdot T)$ have disjoint images. In fact, more is true: if $x \neq \bar{x}$ then the images of $F\left(i_{x}\right) \circ \delta_{1}$ and $F\left(i_{\vec{x}}\right) \circ \delta_{1}$ are disjoint (and similarly if $y \neq \bar{y})$. For, if they were not disjoint, they would continue to fail to be disjoint upon application of the map $\epsilon_{X} \cdot T$. But the diagram

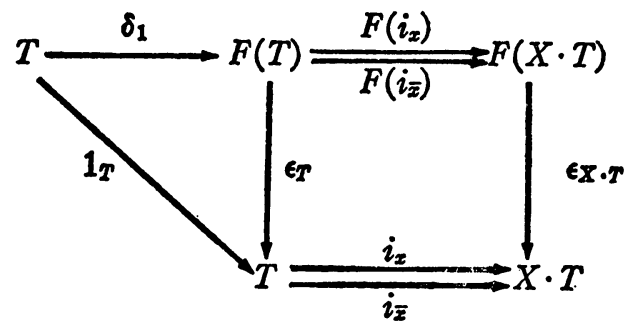

shows that this would be impossible. Hence $k$ is one-to-one, and we have completed the proof that $U$ preserves limits.

Now, in order to show that $U$ has a left adjoint $V$, it suffices to show that for each $(A, \xi)$ there is a family $\left\{B_{\alpha}\right\}$ of sets such that whenever $f:(A, \xi) \rightarrow X \cdot T$, there is a homomorphism $\eta:(A, \xi) \rightarrow B_{\alpha} \cdot T$ and a set map $g: B_{\alpha} \rightarrow X$ such that $f=(g \cdot T) \circ \eta$. But given $f$, its image contains at most $|A|$ elements and thus is contained in $B \cdot T$ where $B \subseteq X$ and $|B| \leqq|A|$. Thus, for a solution set for $(A, \xi)$ we can take the set of all subsets of $A$. This establishes the existence of the component functor $V: a \rightarrow s$, by Freyd's adjoint functor theorem.

Finally we proceed to show that $U$ is monadic by appealing to Beck's Triplability Theorem [1], [3]. Let $d_{0}, d_{1} . X \rightrightarrows Y$ and let $g: Y$ $\rightarrow W$ be coeq $\left(d_{0}, d_{1}\right)$. Suppose that in $a$ we have a diagram

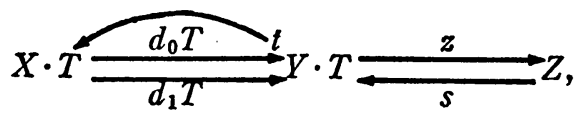

where $\quad z s=\mathrm{id} ., \quad s z=\left(d_{1} \cdot T\right) \circ t, \quad\left(d_{0} \cdot T\right) \circ t=\mathrm{id}$, , and $z \circ\left(d_{0} \cdot T\right)$ $=z \circ\left(d_{1} \cdot T\right)$. Then $z=\operatorname{coeq}\left(d_{0} \cdot T, d_{1} \cdot T\right)$, and in this situation Beck's theorem requires that $Z \cong W \cdot T, z \cong q \cdot T$. These equations are certainly true set-wise, so what we have to show is that the $F$-algebra structure on $Z$ is that of $W \cdot T$. But this is easily seen by observing that $W \cdot T$ does function as the coequalizer of $d_{0} \cdot T$ and $d_{1} \cdot T$ in $a$. This completes the proof of our theorem.

3. Examples. A. Let $M$ be a monoid, let $T$ be a set on which $M$ acts, and let $\operatorname{Mac}(M, T)$ be the category whose objects are sets $A$ on 
which $M$ acts, equipped with $M$-homomorphisms $e_{A}: A \rightarrow T$. A map $f:\left(A, e_{A}\right) \rightarrow\left(B, e_{B}\right)$ is to be an $M$-homomorphism such that $e_{B} \circ f=e_{A}$. $\operatorname{Mac}(M, T)$ is comonadic over $\delta$ by [2]. Then $U(X)=\left(X \times T, e_{X \times T}\right)$ where $m(x, t)=(x, m t)$ and $e_{X \times T}=p_{2}$. The component functor takes $\left(A, e_{A}\right)$ to the set of connected components of $A$ under the action of $M$. (This example was pointed out to the author by John Isbell in a personal communication.)

$B$. Let $a$ be the category of sets equipped with equivalence relations. Using $[a]$ to denote the equivalence class of $a$, define a map $f:(A, \sim) \rightarrow(B, \sim)$ to be a function $f: A \rightarrow B$ such that $f[a]=[\mathrm{fa}]$ for all $a \in A$. Then $Q$ is comonadic, $U(X)=(X,=)$, and $V(A, \sim)=A / \sim$.

C. For a more elaborate example, construct a comonad $(F, \epsilon, \delta)$ as follows. Let $F(A)=A \times 2^{\Lambda}, \epsilon_{A}=p_{1}$, and define $\delta_{A}$ by $\delta_{A}(a, \alpha)$ $=(a, \alpha,\{(b, \alpha) \mid b \in \alpha\})$. Then it is easy to compute that an algebra over this monad is essentially a set $A$ equipped with a function $\phi_{A}: A \rightarrow 2^{A}$ with the property that $\phi_{A}(a)=\phi_{A}\left(a^{\prime}\right)$ whenever $a^{\prime} \in \phi_{A}(a)$. A homomorphism $f:\left(A, \phi_{A}\right) \rightarrow\left(B, \phi_{B}\right)$ is a function such that $f\left(\phi_{A}(a)\right)$ $=\phi_{B}(f(a))$ for all $a \in A$. The terminal object is $T=\{0,1\}$ where $\phi_{T}(0)=\varnothing$ and $\phi_{T}(1)=\{1\}$. The component functor takes $\left(A, \phi_{A}\right)$ to the set

$$
\left\{a \mid a \in A, \phi_{A}(a)=\varnothing\right\} \cup\left\{\phi_{A}(a) \mid a \in A, \phi_{A}(a) \neq \varnothing\right\} .
$$

This case is particularly interesting since it admits another monadic functor $U: S \rightarrow Q$ where $U(A)=(A \Perp\{1\}, \phi), \phi(a)=\{a\}$, and $\phi(1)=\varnothing$. The left adjoint to this functor takes $\left(A, \phi_{A}\right)$ to the set $\left\{\phi_{A}(a) \mid a \in A, \phi_{A}(a) \neq \varnothing\right\}$. Thus, a category can admit reasonable "component functors" other than those for which we have provided a categorical framework.

\section{REFERENCES}

1. R. Davis, Equational systems of functors, Proc. Midwest Category Seminar, Springer, New York, 1967, pp. 92-109.

2. - Universal co-algebra and categories of transition systems, Math. Systems Theory (to appear).

3. E. Manes, A triple miscellany, Dissertation, Wesleyan University, Middletown, Conn., 1967.

4. B. Mitchell, Theory of categories, Academic Press, New York, 1965. MR 34 \#2647.

SoUthern Methodist University 